University of Nebraska - Lincoln

DigitalCommons@University of Nebraska - Lincoln

$6-12-2000$

\title{
Observation of Phase-Matched Relativistic Harmonic Generation
}

Shouyuan Chen

University of Michigan, Ann Arbor, schen6@unl.edu

Anatoly Maksimchuk

University of Michigan, tolya@umich.edu

Eric Esarey

Lawrence Berkeley National Laboratory, Berkeley, California, ehesarey@lbl.gov

Donald P. Umstadter

University of Nebraska-Lincoln, donald.umstadter@unl.edu

Follow this and additional works at: https://digitalcommons.unl.edu/physicsumstadter

Part of the Physics Commons

Chen, Shouyuan; Maksimchuk, Anatoly; Esarey, Eric; and Umstadter, Donald P., "Observation of PhaseMatched Relativistic Harmonic Generation" (2000). Donald Umstadter Publications. 24.

https://digitalcommons.unl.edu/physicsumstadter/24

This Article is brought to you for free and open access by the Research Papers in Physics and Astronomy at DigitalCommons@University of Nebraska - Lincoln. It has been accepted for inclusion in Donald Umstadter Publications by an authorized administrator of DigitalCommons@University of Nebraska - Lincoln. 


\title{
Observation of Phase-Matched Relativistic Harmonic Generation
}

\author{
S.-Y. Chen, ${ }^{1, *}$ A. Maksimchuk, ${ }^{1}$ E. Esarey, ${ }^{2}$ and D. Umstadter ${ }^{1}$ \\ ${ }^{1}$ Center for Ultrafast Optical Science, University of Michigan, Ann Arbor, Michigan 48109 \\ ${ }^{2}$ Center for Beam Physics, Ernest Orlando Lawrence Berkeley National Laboratory, University of California, \\ Berkeley, California 94720
}

(Received 2 July 1999; revised manuscript received 21 March 2000)

\begin{abstract}
Phase-matched relativistic harmonic generation in plasmas is observed for the first time. Thirdharmonic light is detected and discriminated spectrally and angularly from the harmonics generated from competing processes. Its angular pattern is a narrow forward-directed cone, which is consistent with phase matching of a high-order transverse mode in a plasma. The signal level is found to be on the same order of magnitude for a circularly polarized pump pulse as for a linearly polarized pump pulse.
\end{abstract}

PACS numbers: 52.40.Nk, 41.60.-m, 42.65.Ky

It had been predicted theoretically [1,2] and recently verified experimentally that the free electrons in the focus of a high-intensity laser [3] will oscillate relativistically and thus emit harmonics with unique angular patterns [4]. This process, known as relativistic nonlinear Thomson scattering, scatters light into a broad range of angles $\left(\sim 180^{\circ}\right)$ with each lobe having a $>30^{\circ}$ angular width for the low-order harmonics. In this paper we report the experimental observation of the third harmonic emitted into a narrow, hollow cone pointing in the direction of laser propagation. To explain these results, a new theory is presented that describes how phase matching in a plasma can result provided that the harmonic radiation has a highorder transverse structure. The signal level of the harmonic for a circularly polarized pump pulse was found to be on the same order of magnitude as that for a linearly polarized pump pulse, which is characteristically different from harmonic generation from electrons bound to atoms. The increased efficiency due to phase matching might eventually lead to interesting coherent, ultrashort-duration and short-wavelength light sources, in which there is much current interest [5].

Theory for coherent emission in the direction of propagation of the laser beam, referred to as relativistic harmonic generation, has been derived [6,7]. It indicates that-because of the mismatch between the phase velocities of the laser pulse and the generated harmonics and because of the collective response of the plasma-the conversion efficiency should be low unless a means for phase matching, or quasi phase matching [7], is implemented. Experimentally, Liu et al. [8] tried to measure the third-harmonic light produced from relativistic harmonic generation but ultimately could give only an upper limit on the conversion efficiency. Meyer and Zhu [9] claimed to have observed the second relativistic harmonic generated under the condition of beam filamentation. However, such second-harmonic light has been later identified by several groups $[10,11]$ to be associated with the transverse plasma-density depression driven by laser self-channeling or filamentation [12], as is also evident from its broad angular width caused by the plasma density gradient [9].

In this experiment, we used a Ti:sapphire-Nd:glass laser system based on chirped-pulse amplification that produces $\leq 2$-J, 400-fs pulses at $1.053 \mu \mathrm{m}$ wavelength. The $50-\mathrm{mm}$ diam laser beam was beam split into two beams. The first beam was sent to a type-II KDP (potassium dihydrogen phosphate) crystal to produce a second-harmonic pulse which was then focused by an $f / 3$ off-axis parabolic mirror into the center of a gas jet (hydrogen or helium, with a flat top of $750 \mu \mathrm{m}$ in length and a gradient of $250 \mu \mathrm{m}$ ) to preionize the gas into a plasma (i.e., used as a prepulse). The second beam, the pump pulse, was sent through a delay line to vary its temporal delay and then through a half-wave wave plate and a thin-film polarizer to vary its energy. After that, it was focused by an $f / 3.3$ lens onto the same gas jet with its focus overlapping with, and its direction collinearly counterpropagating with respect to, that of the second-harmonic prepulse. The prepulse has a focal spot of $12 \mu \mathrm{m}$ FWHM (full width at half maximum), and the pump pulse has a focal spot of $14 \mu \mathrm{m}$. In this experiment, the prepulse energy was fixed at $\sim 90 \mathrm{~mJ}$, corresponding to a peak vacuum intensity of $2 \times 10^{17} \mathrm{~W} / \mathrm{cm}^{2}$, which is 3 orders of magnitude higher than the ionization threshold [13] of hydrogen, enough to produce a plasma column of $>50 \mu \mathrm{m}$ diameter. The pump-pulse intensity was varied by adjusting the half-wave wave plate and the maximum was about $2 \times 10^{17} \mathrm{~W} / \mathrm{cm}^{2}$, which corresponds to a normalized vector potential $a_{0}=$ $8.5 \times 10^{-10} \lambda[\mu \mathrm{m}] I^{1 / 2}\left[\mathrm{~W} / \mathrm{cm}^{2}\right]=0.4$. The polarization of the prepulse was set to be linearly polarized, and that of the pump pulse was controlled to be either linearly polarized or circularly polarized by use of a quarter-wave wave plate located before the focusing lens. For the data presented below, the pump pulse was linearly polarized.

The third-harmonic light generated by the pump pulse and emitted in the forward direction was collected by the focusing parabolic mirror of the prepulse and was 
then separated from the prepulse and the pump pulse by passing through 527-nm-wavelength and 1053-nmwavelength high-reflection dielectric mirrors. It was then diagnosed with an imaging grating spectrometer and a beam-pattern imaging system. The use of a prepulse at the second harmonic is to make sure that it cannot produce harmonics at the wavelength of third harmonic of the pump pulse. The delay of the pump pulse was set such that the pump pulse entered the plasma 7 ps after the prepulse passing through the gas jet, avoiding the mixing of the prepulse and the pump pulse in the plasma to generate the third harmonic but not allowing enough time for recombination. No significant change in the third-harmonic signal was observed when the delay was varied from 5 to $20 \mathrm{ps}$.

Figure 1 shows the spectra of the third harmonic generated in helium at a pump intensity of $1.5 \times 10^{17} \mathrm{~W} / \mathrm{cm}^{2}$ and a gas density of $3 \times 10^{19} \mathrm{~cm}^{-3}$ with and without the prepulse. As can be seen, the spectrum without the prepulse contains two spectral components: the first one is strong, narrow $(\sim 8 \AA)$, and peaked close to the exact third-harmonic wavelength, and the second one is weaker, broad $(\sim 60 \AA$ ), and blueshifted (by $\sim 25 \AA$ ). A similar spectrum was observed for hydrogen. When the prepulse was turned on, the intensity of the narrow unshifted component remained pretty much the same while the broad blueshifted component disappeared, for both the cases of helium and hydrogen.

The broad blueshifted component is consistent with the third harmonic generated from ionization [14], because it disappears when the gas is preionized and the broadening and blueshifting are consistent with ionization $[8,15]$. We believe that the narrow unshifted component is the third harmonic produced from relativistic harmonic generation because its spectrum is narrow (about $1 / 3$ of the bandwidth of the pump pulse) and unshifted and its spectrum

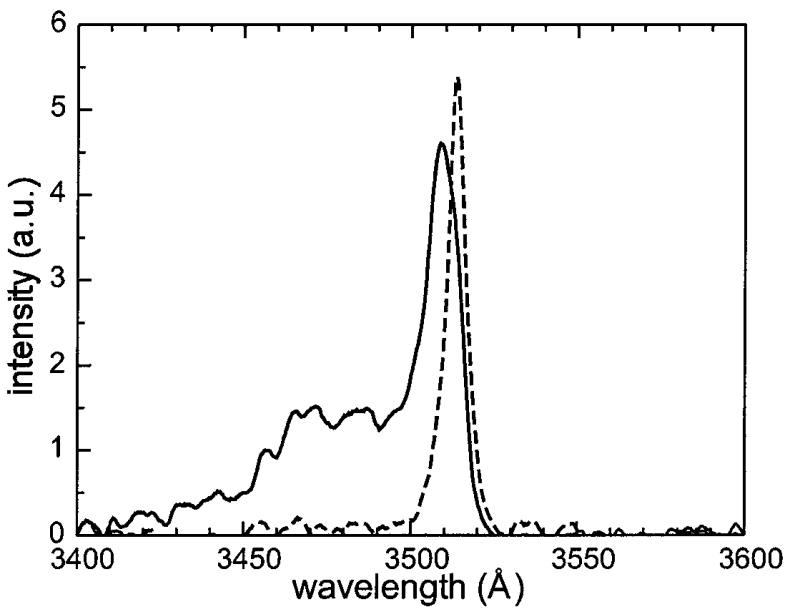

FIG. 1. Spectra of the third harmonic generated in helium for $3 \times 10^{19} \mathrm{~cm}^{-3}$ gas density and $1.5 \times 10^{17} \mathrm{~W} / \mathrm{cm}^{2}$ pump laser intensity with (dashed line) and without (solid line) the prepulse. and intensity do not change significantly when the prepulse is turned on (since the foot of the pump pulse is enough to fully ionize the gas and turn it into a plasma). In addition, the narrow spectral width $(8 \AA)$ observed is strong evidence to support that this third harmonic was generated from a coherent and phase-matched process, as compared to the broad spectral bandwidth (120 $\mathrm{A})$ of the third harmonic generated from incoherent relativistic Thomson scattering [4].

The intensity of the narrow unshifted spectral component as a function of plasma electron density $N_{e}$ for a fixed laser vacuum intensity is plotted in Fig. 2(a) and as a function of pump laser intensity for a fixed plasma density in Fig. 2(b), for both helium and hydrogen with the prepulse. As shown, the intensity of this unshifted signal scales as $I_{0}^{3}$ (where $I_{0}$ is the pump intensity), which is consistent with relativistic third-harmonic generation. When the gas density was varied, the intensity of this third harmonic varied
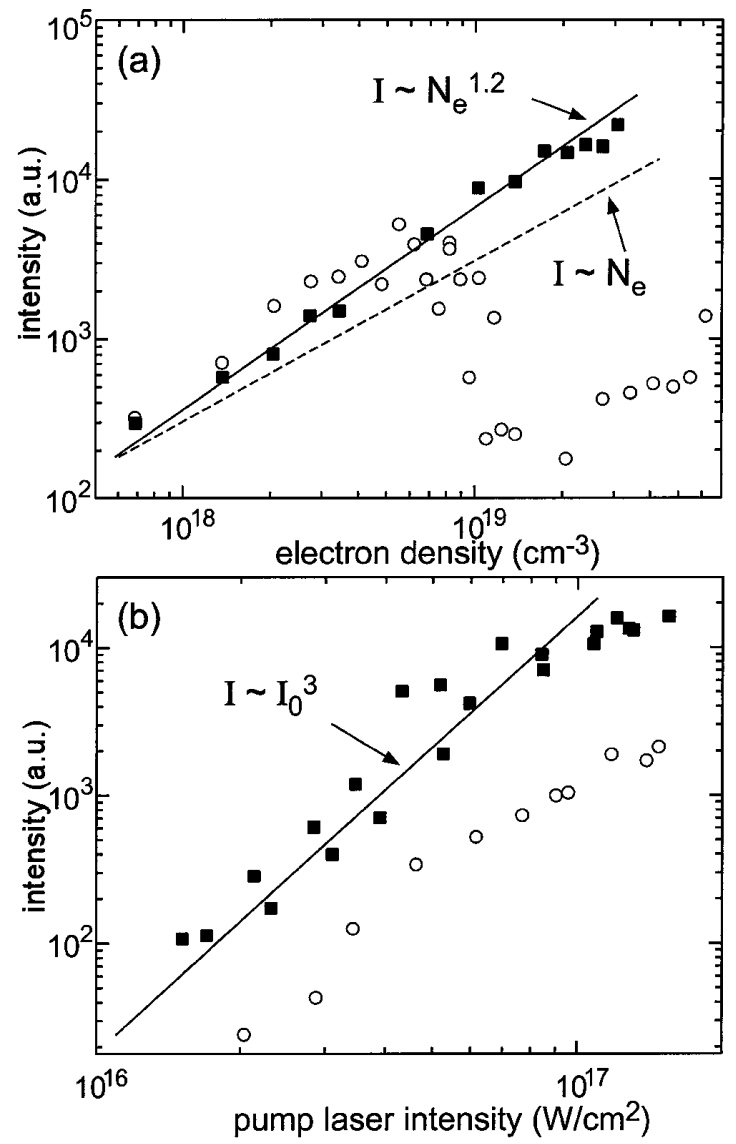

FIG. 2. Intensity of the narrow unshifted third harmonic in helium (open circles) and in hydrogen (solid squares) as a function of plasma electron density and laser intensity when a prepulse is used. (a) shows the dependence of the intensity on electron density for a laser vacuum intensity of $1.5 \times 10^{17} \mathrm{~W} / \mathrm{cm}^{2}$ and (b) shows the dependence of the intensity on pump laser intensity for a plasma electron density of $8 \times 10^{18} \mathrm{~cm}^{-3}$ for helium and $3 \times 10^{19} \mathrm{~cm}^{-3}$ for hydrogen. Solid and dashed lines are plotted to help view the scaling of the signal. 
as $N_{e}^{1.2}$ for hydrogen. For the case of helium, the signal intensity at low densities increases with increase of gas density in the same way as for hydrogen. However, it starts to decrease with increase of gas density when the gas density is higher than $3 \times 10^{18} \mathrm{~cm}^{-3}$ and thus becomes 2 orders of magnitude lower than that for the case of hydrogen at a gas density of $3 \times 10^{19} \mathrm{~cm}^{-3}$. This is believed to be a result of the decrease of the peak pump intensity due to ionization defocusing of the pump pulse at high gas densities [16] (note that the ionization threshold for the second electron in a helium atom is $10^{16} \mathrm{~W} / \mathrm{cm}^{2}$ so that the prepulse may not fully ionize the helium at near the entrance of the pump pulse, especially because it is also ionization defocused at the opposite end of the gas jet at high densities). More importantly, the intensity of this thirdharmonic light generated in helium is about the same as that generated in hydrogen for the same plasma electron density (before the refractive defocusing starts to affect the helium results). This supports the argument for relativistic harmonic generation which depends on the free-electron density only and not the type of gas.

The parabolic mirror collimated the forwardpropagating third-harmonic beam and converted the angular pattern of this emission into the transverse intensity pattern of the collimated beam. Therefore, by imaging the latter with a CCD (charge-coupled device) camera and matching narrow bandpass filters, we obtained the angular profile of the third harmonic. Figure 3 shows the angular pattern of the narrow unshifted third-harmonic signal for a hydrogen gas density of $1.5 \times 10^{19} \mathrm{~cm}^{-3}$ and a pump laser intensity of $1.7 \times 10^{17} \mathrm{~W} / \mathrm{cm}^{2}$ with the prepulse. It shows a cone with a radius (cone angle) of $5.62^{\circ}$ and a ring width of $<0.44^{\circ}$. The narrow angular width $\left(<0.44^{\circ}\right)$

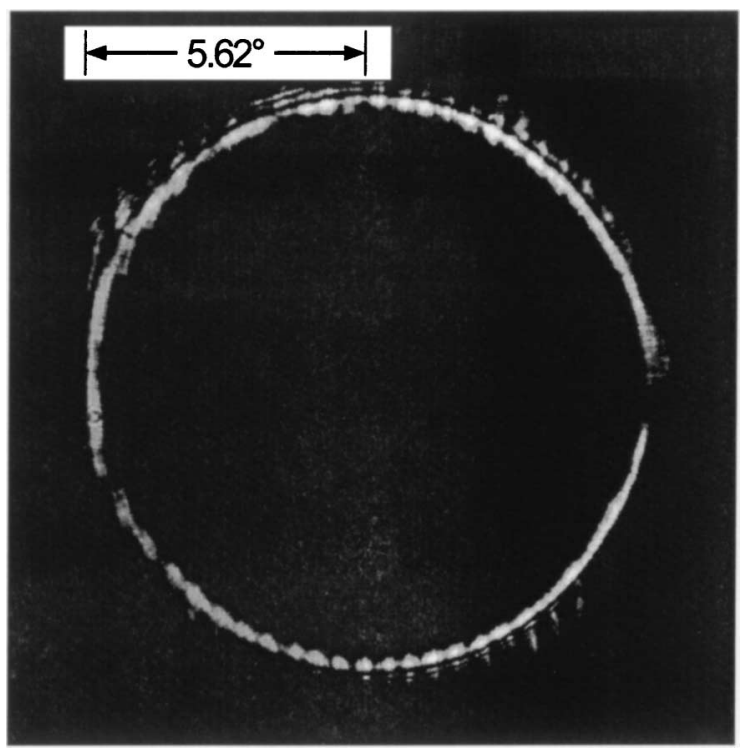

FIG. 3. Angular pattern of the third harmonic generated for a plasma density of $3 \times 10^{19} \mathrm{~cm}^{-3}$ and a pump laser intensity of $1.7 \times 10^{17} \mathrm{~W} / \mathrm{cm}^{2}$, when a prepulse is used. of the cone is strong evidence for good coherence (phase matching) of this third-harmonic beam, as compared to the broad angular width $\left(>30^{\circ}\right)$ of the angular pattern of incoherent harmonic emission from single-particle relativistic Thomson scattering [4]. Furthermore, the angular pattern of the broad blueshifted third harmonic was observed to have a Gaussian profile pointing in the direct forward direction, similar to that of the transmitted pump beam. This also helps discriminate between these two third-harmonic signals.

Regardless of the details of the mechanism driving the harmonic, the harmonic amplitude will be greatly enhanced provided that the phase-matching condition $\Delta \phi=0$ is satisfied, where $\Delta \phi \simeq k_{3} z^{\prime}-3 k_{0} z, \quad z$ $\left(z^{\prime}\right)$ is along the direction of propagation of the pump (third harmonic), $z=z^{\prime} \cos \theta, \quad \theta$ is the cone angle of the third harmonic, and $k_{0}\left(k_{3}\right)$ is the axial wave number of the pump (third harmonic) along the propagation direction. In terms of the index of refraction $\eta_{0,3}=c k_{0,3} / \omega_{0,3}$, the phase-matching condition yields $\cos \theta=\eta_{3} / \eta_{0}$. Near the focus the index of refraction associated with a Laguerre-Gaussian mode [17] is $\eta^{2}=1-\omega_{p}^{2} / \gamma \omega^{2}-4(2 m+p+1) c^{2} / r_{s}^{2} \omega^{2}$, where $\omega=2 \pi c / \lambda$ is the mode frequency, $\omega_{p}=2 \pi c / \lambda_{p}=$ $5.64 \times 10^{4} \sqrt{N_{e}\left[\mathrm{~cm}^{-3}\right]}$ is the plasma frequency, $r_{s}$ is the spot size, $\gamma=\sqrt{1+a_{0}^{2} / 2}$, and $m$ and $p$ are, respectively, the radial and poloidal mode numbers. The third harmonic is clearly a very high order mode, and we will approximate $4(2 m+p+1) / r_{s}^{2} \simeq 4 / r_{3}^{2}$ with $r_{3}^{2} \ll r_{0}^{2}$, where $r_{3}$ is the transverse width of the third-harmonic ring. In terms of the measured angular width of the ring $\Delta \theta, r_{3} \simeq \Delta \theta Z_{R} / 4$, where $Z_{R}=\pi r_{0}^{2} / \lambda_{0}$ is the Rayleigh length of the pump. Hence, the phase-matching condition can be written as

$$
\cos \theta=\frac{\left(1-\lambda_{0}^{2} / \gamma n^{2} \lambda_{p}^{2}-\lambda_{0}^{2} / \pi^{2} n^{2} r_{3}^{2}\right)^{1 / 2}}{\left(1-\lambda_{0}^{2} / \gamma \lambda_{p}^{2}-\lambda_{0}^{2} / \pi^{2} r_{0}^{2}\right)^{1 / 2}},
$$

where $n=3$ is the harmonic number.

In the $1 \mathrm{D}$ limit, $\eta_{3}>\eta_{0}$ and phase matching is not possible. In fact, the detuning length [6] is $Z_{D} \simeq 3 \lambda_{p}^{2} / 8 \lambda \sim 40 \mu \mathrm{m} \ll Z_{R}$. This is contrary to harmonic generation in rare gases, in which the interaction distance is limited by diffraction and not detuning. In $3 \mathrm{D}$, phase matching is possible if $r_{3}^{2} \ll r_{0}^{2}$, which is the case in the present experiments. We shall assume a simple form $r_{3}^{2} \simeq\left(r_{0}^{2} / 3\right) \epsilon^{2} N_{0} / N_{e}$, where $\epsilon^{2} \ll 1$ and $N_{0}$ is a normalization density $\left(N_{0}=10^{19} \mathrm{~cm}^{-3}\right)$, since $\Delta \theta$ is observed to decrease with increasing density $N_{e}$. For the parameters of the experiment, $N_{e}=10^{19} \mathrm{~cm}^{-3}$ $\left(\lambda_{p}=11 \mu \mathrm{m}\right)$ and $r_{0}=12 \mu \mathrm{m}$, we find $\theta=5.3^{\circ}$ and $\Delta \theta=0.42^{\circ}$ for $\epsilon=0.12$, which are in approximate agreement with the measured values $\left(\theta=5.62^{\circ}\right.$ and $\delta \theta=0.44^{\circ}$ ). Figure 4 shows the angle of the cone as a function of plasma density, and the inset shows the prediction of Eq. (1) with $\epsilon=0.12$. Both show a similar 


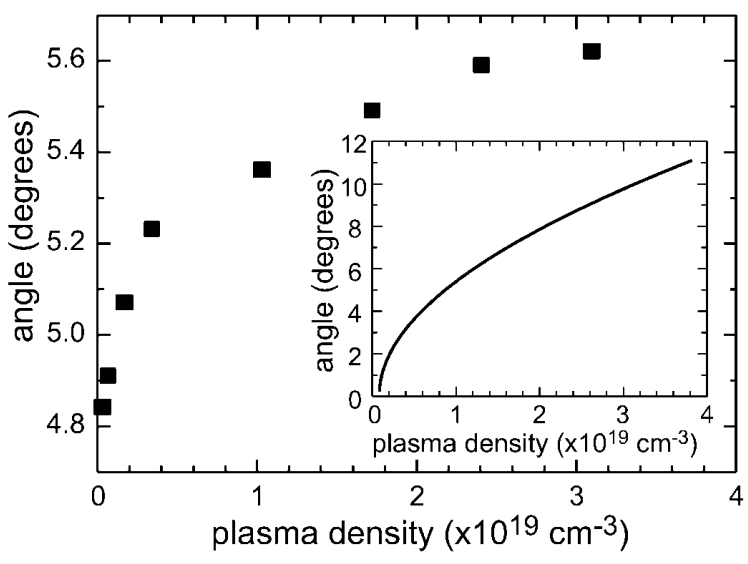

FIG. 4. Angle (radius) of the cone of emission for the narrow unshifted third harmonic as a function of plasma density at a laser intensity of $1.7 \times 10^{17} \mathrm{~W} / \mathrm{cm}^{2}$. The error is indicated by the size of the symbols. The inset shows the theoretical prediction that uses Eq. (1).

trend of increase of angle with increasing plasma density, although their details are different. In addition, the cone angle was observed to be roughly independent of laser intensity, also consistent with theory. The discrepancy in Fig. 4 is believed to be caused by the approximate manner in which the Gouy phase shifts are treated in Eq. (1), as well as an uncertainty in modeling the transverse structure of the third harmonic.

For a gas, the linear dispersion is orders of magnitude less than that for a plasma of equal density. Equation (1) applies only if the 1D dephasing length is shorter than the interaction length, which is not the case for a gas. For a gas, the harmonic cone angle should be nearly zero and the variation with gas density should be negligible. Furthermore, we found that the cone angle is the same for helium as for hydrogen at the same plasma density. Therefore, these observations are consistent with relativistic harmonic generation from plasmas and rule out the possibility of originating from atomic effects.

The absolute energy conversion efficiency for this thirdharmonic beam for a linearly polarized pump pulse at a plasma density of $3 \times 10^{19} \mathrm{~cm}^{-3}$ and a laser intensity of $1.7 \times 10^{17} \mathrm{~W} / \mathrm{cm}^{2}$ is $\sim 2 \times 10^{-5}$, obtained by integrating the cone image taken with the CCD camera. This efficiency is 2 orders of magnitude higher than the predicted saturation efficiency $\left(\sim 4 \times 10^{-8}\right)$ without phase matching in the directly forward direction in the theory derived by Esarey et al. [6]. This is to be expected since in the latter case the detuning length is only $14 \mu \mathrm{m}$, much shorter than the length of the gas jet we used.

All of the results for the narrow unshifted third-harmonic component are basically the same for a circularly or a linearly polarized pump pulse. The polarization of the third harmonic was found to be the same as that of the pump laser for both linear and circular polarization, for every portion of the cone. The only difference is that the intensity of the third harmonic is somewhat lower for a circularly polarized pump; however, the signal levels are still the same order of magnitude. This is characteristically different from third-harmonic generation due to atomic nonlinear susceptibility, for which circular polarization led to undetectable signals compared to that from linear polarization $[8,18]$. These observations imply that some nonideal plasma effect, such as small-scale density modulations resulting from Raman backscatter, is likely to be important in the underlying harmonic generation mechanism. A self-consistent theory of phase-matched harmonic generation in plasmas is the subject of ongoing research.

S.-Y.C. and A. M. were supported by the National Science Foundation, D. U. by the Division of Chemical Sciences, Office of Basic Energy Sciences, Office of Energy Research, U.S. Department of Energy, and E.E. by the Division of High Energy Physics, U.S. Department of Energy. We thank G. Shvets for useful discussions.

*Present address: Institute of Atomic and Molecular Sciences, Academia Sinica, P.O. Box 23-166, Taipei 106, Taiwan.

[1] Vachaspati, Phys. Rev. 128, 664 (1962); L. S. Brown and T.W. B. Kibble, Phys. Rev. 133, A705 (1964); E. S. Sarachik and G. T. Schappert, Phys. Rev. D 1, 2738 (1970); C. I. Castillo-Herrera and T. W. Johnston, IEEE Trans. Plasma Sci. 21, 125 (1993).

[2] E. Esarey, S. K. Ride, and P. Sprangle, Phys. Rev. E 48, 3003 (1993).

[3] P. Maine et al., IEEE J. Quantum Electron. 24, 398 (1988).

[4] S.-Y. Chen, A. Maksimchuk, and D. Umstadter, Nature (London) 396, 653 (1998).

[5] Special issue on "Nonlinear Optics with Free Electrons," edited by D. Umstadter and T. Norris [IEEE J. Quantum Electron. 33, 1878-1968 (1997)].

[6] E. Esarey et al., IEEE Trans. Plasma Sci. 21, 95 (1993); W. B. Mori, C. D. Decker, and W. P. Leemans, ibid. 21, 110 (1993).

[7] J. M. Rax and N. J. Fisch, IEEE Trans. Plasma Sci. 21, 105 (1993).

[8] X. Liu, D. Umstadter, E. Esarey, and A. Ting, IEEE Trans. Plasma Sci. 21, 90 (1993).

[9] J. Meyer and Y. Zhu, Phys. Fluids 30, 890 (1987).

[10] K. Krushelnick et al., Phys. Rev. Lett. 75, 3681 (1995).

[11] V. Malka et al., Phys. Plasmas 4, 1127 (1997).

[12] D. S. Bethune, Phys. Rev. A 23, 3139 (1981).

[13] S. Augst et al., Phys. Rev. Lett. 63, 2212 (1989).

[14] X.F. Li et al., Phys. Rev. A 39, 5751 (1989); F. Brunel, J. Opt. Soc. Am. B 7, 521 (1990).

[15] C. W. Siders et al., J. Opt. Soc. Am. B 13, 330 (1996).

[16] A. J. Mackinnon et al., Phys. Rev. Lett. 76, 1473 (1996).

[17] E. Esarey and W.P. Leemans, Phys. Rev. E 59, 1082 (1999).

[18] J.F. Ward and G. H. C. New, Phys. Rev. 185, 57 (1969). 